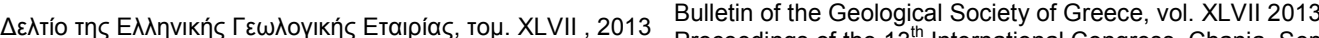

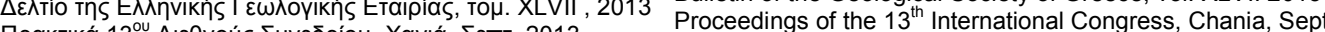

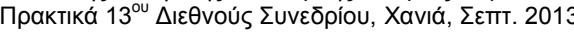
2013

\title{
MULTIDIMENSIONAL EARTHOUAKE FREOUENCY DISTRIBUTIONS CONSISTENT WITH NON-EXTENSIVE STATISTICAL PHYSICS: THE INTERDEPENDENCE OF MAGNITUDE, INTEREVENT TIME AND INTEREVENT DISTANCE IN NORTH CALIFORNIA
}

\author{
Tzanis A. ${ }^{1}$, Vallianatos . $^{2}$ and Efstathiou A. ${ }^{1}$ \\ ${ }^{I}$ National and Kapodistrian University of Athens, Faculty of Geology and Geoenvironment, \\ Department of Geophysics and Geothermy,atzanis@geol.uoa.gr,aefstathiou@geol.uoa.gr \\ ${ }^{2}$ Laboratory of Geophysics and Seismology, Technological Educational Institute of Crete, \\ Chania, GR 73133, Crete, Greece,fvallian@chania.teicrete.gr
}

\begin{abstract}
It is now accepted that the active tectonic grain comprises a self-organized complex system, therefore its expression (seismicity) should be manifested in the temporal and spatial statistics of energy release rates, and exhibit memory due to long-range interactions in a fractal-like space-time. Such attributes can be properly understood in terms of Non-Extensive Statistical Physics. In addition to energy release rates expressed by the magnitude $M$, measures of the temporal and spatial interactions are the time $(\Delta t)$ and hypocentral distance $(\Delta d)$ between consecutive events. Recent work indicated that if the distributions of $M, \Delta t$ and $\Delta d$ are independent so that the joint probability $p(M, \Delta t, \Delta d)$ factorizes as $p(M) p(\Delta t) p(\Delta d)$, earthquake frequency is related to $M, \Delta t$ and $\triangle d$ by well defined power-laws consistent with NESP. The present work applies these concepts to investigate the self-organization and temporal/spatial dynamics of North Californian seismicity. The results indicate that the statistical behaviour of seismicity in this area is consistent with NESP predictions and has attributes of universality, as its holds for a very broad range of spatial, temporal and magnitude scales. They also indicate that the expression of the regional active tectonic grain comprises a mixture of processes significantly dependent on $\Delta d$, which include near $(<100 \mathrm{~km})$ and far $(>400 \mathrm{~km})$ field interactions.
\end{abstract}

Key words: Tsallis entropy, complexity, non-extensivity, statistical seismology.

\section{Пєрí $\lambda \psi \eta$}

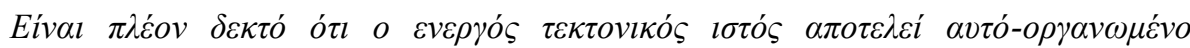

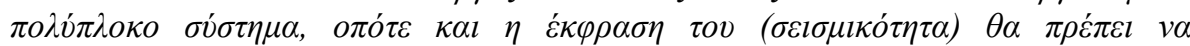

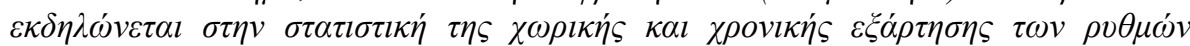

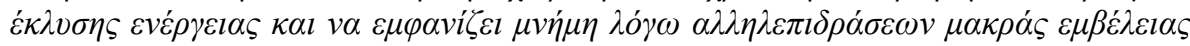

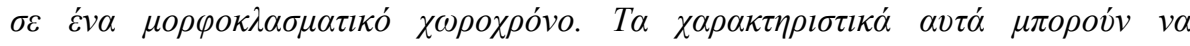

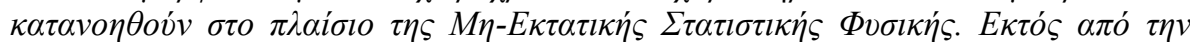

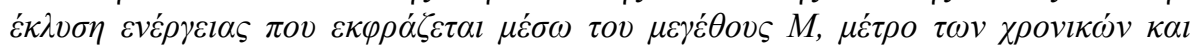

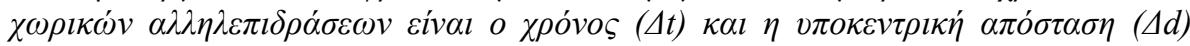

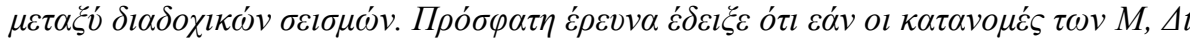

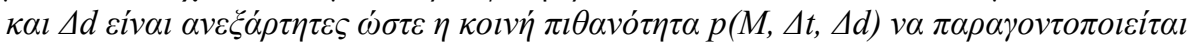

XLVII, No $3-1326$ 


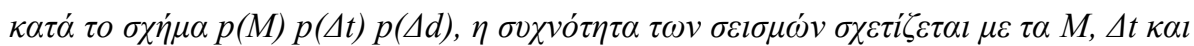

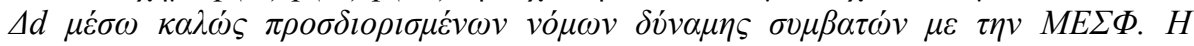

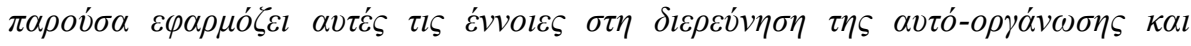

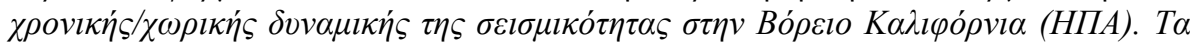

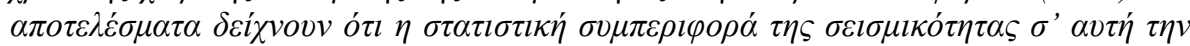

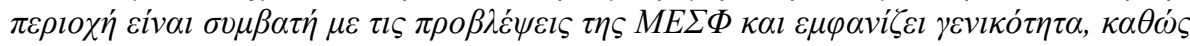

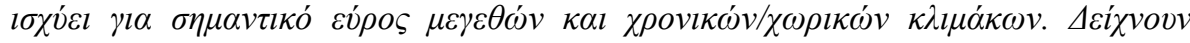

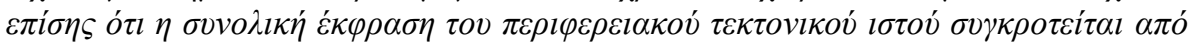

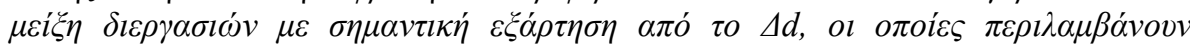
$\alpha \lambda \lambda \eta \lambda \varepsilon \pi l \delta \rho \alpha \dot{\sigma \varepsilon l \varsigma} \varepsilon \gamma \gamma \dot{\varsigma} \varsigma(<100 \mathrm{~km}) \kappa \alpha l \mu \alpha \kappa \rho \alpha \dot{v}(>400 \mathrm{~km}) \pi \varepsilon \delta i o v$.

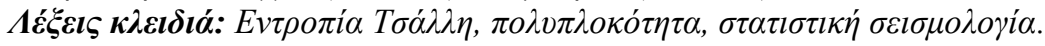

\section{Introduction}

The long term evaluation of earthquake hazard in terms of the regional statistical properties of earthquake occurrence and recurrence is usually based on the celebrated frequency - magnitude (F-M) distribution of Gutenberg and Richter (1944). This states that the frequency of earthquake occurrence is related to earthquake magnitude via a simple relationship of the form $\log N=a-b M$, where $N$ is the number of earthquakes in a specified time interval. Barring the complications arising from studies of the F-M distribution on the global scale, the standard assumption about seismogenesis on regional and local scales was that it basically comprises a random (hence memoryless) extensive process and that individual events are uncorrelated, at least at the higher magnitude range. The Gutenberg - Richter F-M distribution was understood in this context and the standard tools currently used for earthquake hazard analysis are all based on this premise. Thus, it was generally accepted that seismicity (seismogenesis) obeys Boltzmann-Gibbs dynamics, although an apparent contradiction in that viewpoint is that the F-M distribution implies scale-free grading of frequency and magnitude, which cannot be derived from the Boltzmann-Gibbs formalism.

The concepts of fractal geometry, self-organized criticality and complexity introduced in the 1980's and 1990's have gradually shifted the paradigm. It is now accepted that the active tectonic grain comprises a complex dynamic system, the general understanding being that it is critical (e.g. Bak and Tang, 1989; Sornette and Sornette, 1989; many others since). A sizeable minority point of view proposes alternative complexity mechanisms, as for instance is the Epidemic-Type Aftershoc k Sequences model (e.g. Ogata, 1988), the Coherent Noise Model (Celikoglu et al, 2010), the Fertility Heterogeneity model (Saichev and Sornette, 2012), etc. At any rate, dynamic complex systems evolving in a fractal-like space-time are characterized by long-range interactions and longterm memory. This, in turn, implies that the statistics of earthquake occurrence, at least on a regional scale, should be manifested in certain traits of the F-M distribution and of all analogous frequency distributions pertaining to its temporal and spatial dependence. Specifically, one should expect power laws resulting from the tails generated by the long-term memory of the system.

The presently accepted interpretation of the Gutenberg - Richter F-M distribution is that it expresses the scale-free statistics of a fractal active tectonic grain; with relationships between the constant $b$ and the fractal dimension of the tectonic grain having been derived in various ways. However, the F-M distribution is static and says nothing about the temporal and spatial dynamics of the seismogenetic system. A measure of the temporal dynamics is the time lapsed between consecutive events above a magnitude threshold over a given area: this parameter is variably referred to as interevent time, waiting time, calm time etc. Understanding the statistics of the earthquake frequency - interevent time (F-T) distribution is obviously key to understanding the seismogenetic system and has been studied by several researchers. The empirical F-T distributions generally exhibit power-law behaviour and long tails and have been investigated with standard statistical models with tails, reducible to power laws in some way or another (e.g. gamma distribution, Weibull distribution etc.). Several investigators have applied the gamma distribution and have suggested

$\underline{\text { XLVII, No } 3-1327}$ 
universality analogous to the Gutenberg - Richter law (e.g. Corral, 2004), to which other investigators object on the premise of model-based studies, suggesting that the interevent times are mixed distributions of correlated aftershock and uncorrelated background processes (e.g. Touati et al, 2009). At any rate, these studies have generally been empirical or model-based; in the latter case, the underlying background process has always been conjectured to obey extensive thermodynamics (i.e. to be random). A measure of the spatial dependence is the hypocentral distance between consecutive events above a magnitude threshold over a given area (interevent distance). With less than a handful of direct (e.g. Abe and Suzuki, 2003) or indirect treatises, (e.g. Baiesi and Paczucki, 2004; Marsan and Legliné, 2008), the properties and statistics of earthquake frequency - interevent distance (F-D) distribution is practically terra incognita.

The most recent development in the statistical description of earthquake occurrence is the introduction of the concept of non-extensivity. A simple argument for this point of view is that if the frequency distributions of the relevant parameters exhibit the traits expected of non-extensive systems (power laws), then it is very likely that the system generating these distributions is universally non-extensive and not a mixture of extensive, or extensive and non-extensive processes, as the contesting view proposes. The Non-Extensive Statistical Physics (NESP) approach has attracted growing attention during the past few years (e.g. Vallianatos and Telesca, 2012) with several researchers studying the properties of the F-M and F-T distributions. However, if the seismogenetic process is indeed complex and long-range/long-memory, then the size, time and spatial statistics of earthquakes should be not be unrelated! Insofar as earthquakes occur by the triggering of successor events within the temporal and spatial interaction radius of predecessor events, (which depends on their size), or by long-range effects in the correlated space of the seismogenetic system, the frequency of earthquakes should be related to the magnitude, the interevent time and the interevent distance by multidimensional extensions of the one-dimensional power-laws predicted by the complexity model describing the system, in this case the NESP formalism.

The present paper marks the beginning of a systematic attempt to verify the conjecture and assist in validating/refuting the NESP approach to seismicity by studying the local and regional characteristics of different seismogenetic areas of the world, starting with Northern California (United States). The presentation will focus on the analysis of bivariate F-M-T distributions, while using the interevent distances as spatial constraints or spatial filters for studying the spatial dependence of the energy and time dynamics of North Californian seismicity. It shall be demonstrated that the frequency of earthquake occurrence at Northern California is multiply related to the magnitude, the interevent time by means of well defined multi-dimensional power-laws consistent with NESP and has attributes of universality, as its holds for a very broad range of spatial, temporal and magnitude scales. It shall also be demonstrated that the expression of the regional active tectonic grain (seismicity) is produced by an unexpectedly complex system comprising a mixture of processes that depend significantly on the distance between earthquakes.

\section{Non-Extensive Statistical Physics}

\subsection{Tsalis Entropy and Probability Distribution}

The context of Non-Extensive Statistical Physics (NESP), originally introduced by Tsallis (1988, 2009), has recently been suggested to comprise an appropriate tools for the analysis of complex dynamic systems with scale invariance, long-range interactions, long-range memory and systems that evolve in a fractal-like space-time (e.g. Gell-Mann and Tsallis, 2004).

Let $X$ be a seismicity parameter (e.g. energy, interevent time etc.) and $p(X) d X$ the probability of finding its value in $[X, X+d X]$, such that $\int_{W} p(X) d X=1$, where $W$ is the support of $X$ and expresses the total number of microscopic configurations of the system. Non-equilibrium states in systems with complex behaviour can be described by the Tsallis (1988) entropic functional:

XLVII, No $3-1328$ 
$S_{q}=k \frac{1}{q-1}\left[1-\int_{W} p^{q}(X) d X\right]$,

where $k$ is the Boltzmann constant and $q$ the entropic index. The latter is a measure of the non-exte nsivity of the system and for the particular case $q=1$ Eq. 1 reduces to the Boltzmann-Gibbs entrop y $S_{B G}=-k \int_{W} p(X) \ln (p(X)) d X$.

The Tsallis entropy shares properties with the Boltzmann-Gibbs entropy, including concavity and fulfilment of the H-theorem. However, it is pseudo-additive in the sense that it is not proportional to the number of the elements of the system as is $S_{B G}$. Thus, for a composite of two statistically independent systems, $A$ and $B, S_{q}(A, B)=S_{q}(A)+S_{q}(B)+(1-q) S_{q}(A) S_{q}(B)$, leading to superadditivity for $q<1$, additivity for $q=1$, i.e. Gibbs-Boltzmann statistics, and sub-additivity for $q>1$.

Maximization of the Tsallis entropy yields the q-exponential distribution

$p(X)=\frac{1}{Z_{q}} \exp _{q}\left\lfloor-\frac{\lambda}{I_{q}}\left(X-\langle X\rangle_{q}\right)\right\rfloor$,

where $\langle X\rangle_{q}=\int_{W} X p_{q}(X) d X$ is the $q$-expectation value of $X, \lambda$ is an appropriate Lagrange multiplier, $Z_{q}=\int_{W} d X \exp _{q}\left\lfloor-\lambda \cdot I_{q}^{-1} \cdot\left(X-\langle X\rangle_{q}\right)\right\rfloor$ is a generalized canonical partitioning function, and $I_{q}=\int_{W} d X[p(X)]^{q}$. In Eq. 2, the function $\exp _{q}($.$) represents the q$-exponential function

$\exp _{q}(X)=\left\{\begin{array}{ll}(1+(1-q) X)^{\frac{1}{1-q}} & 1+(1-q) X>0 \\ 0 & 1+(1-q) X \leq 0\end{array}\right.$,

and comprises a generalization of the exponential function: for $q=1, \exp _{1}(X)=\mathrm{e}^{x}$. As evident from Eq. 2 and Eq. 3, the probability $p(X)$ is a power-law with a long tail if $q>1$, corresponding to $s u b$ additivity, an exponential distribution if $q=1$, corresponding to additivity, and a cut-off if $0<q<1$, corresponding to super-additivity; the cut-off appears at $X_{\mathrm{c}}=X_{0}(1-q)^{-1}$, with $X_{0}=(1-q) \cdot\left\langle X_{q}\right\rangle+\lambda / I_{q}$.

If the empirical distribution of $X$ (i.e. the escort probability) is $P_{q}(X)$, then the cumulative probability function (CDF) derived from the above analysis is:

$P(>X)=\int_{X}^{\infty} d X P_{q}(X)$.

In the case of $q>1$ and $X \in[0, \infty)$, the CDF becomes:

$P(>X)=\int_{X}^{\infty} d X P_{q}(X) \ldots \Rightarrow \ldots P(>X)=\exp _{q}\left(-\frac{X}{X_{0}}\right)=\left[1-(1-q)\left(\frac{X}{X_{0}}\right)\right]^{\frac{1}{1-q}}$.

which is a q-exponential distribution. When $q>1$ the CDF exhibits a long tail, which becomes longer with increasing $q$; the system experiences long-range correlations and has long-term memory. When $q=1$, the q-exponential distribution reduces to the common exponential distribution: the system is a random process (uncorrelated and memory-less). When $q<1$, the distribution exhibits a cut-off, i.e. $P(>\mathrm{X})=0$ whenever the argument becomes negative and is characterized by a bounded correlation radius.

\subsection{NESP Distribution Law for Earthquake Magnitude and Interevent Time}

The frequency-magnitude distribution has been approximated with a number of NSEP-compatible models (Sotolongo-Costa and Posadas, 2004; Silva et al., 2006; Telesca 2011, 2012 etc.). These all consider the interaction of two rough fault walls (asperities) and the fragments filling space between them, which is supposed to modulate earthquake triggering (the fragment-asperity model). However, they differ in their assumption of how the total energy stored in the asperities and frag-

$\underline{\text { XLVII, No } 3 \text { - } 1329}$ 
ments scales with their linear characteristic dimension. Herein, we propose that the assumption made by Telesca $(2011,2012)$, that the energy scales with the area of the fragments and asperities $\left(E \propto r^{2}\right)$ so that $M=2 / 3 \log (E)$, is representative of the seismogenetic process. Accordingly, we adopt his model, in which the cumulative frequency-magnitude distribution reads:

$P(>M)=\log \left(\frac{N(>M)}{N_{M=0}}\right)=\left(\frac{2-q_{M}}{1-q_{M}}\right) \log \left(1-\left[\frac{1-q_{M}}{2-q_{M}}\right]\left[\frac{10^{M}}{\alpha^{2 / 3}}\right]\right)$

This relationship describes from first principles and in NESP formalism, the cumulative distribution of the number of earthquakes $N$ with magnitude greater than a threshold $M$ in a seismic region, normalized by the total number of earthquakes. The constant $\alpha$ expresses the proportionality between the released energy $E$ and the fragment size $r$ and $q_{M}$ is the entropic index.

The CDF expressed by Eq. 4 is the only NESP formulation proposed for the one-dimensional distributions F-T (Abe and Suzki, 2005) and F-D (Abe and Suzuki, 2003). The empirical application of NESP to interevent times has been taken up by a handful of authors. Abe and Suzuki (2005) investigated the temporal properties of the seismicity in California and Japan; Carbone et al (2005) investigated the Italian seismicity; more recently, Vallianatos et al. (2012) investigated the spatiotemporal properties of the 1996 Aigion (Greece) aftershock sequence, Vallianatos et al. (2013) the temporal behaviour of the 2011-2012 seismicity crisis in the Santorini volcanic complex (Greece) and Vallianatos and Sammonds (2013) the behaviour of global seismicity prior to the 2004 Sumatran and 2011 Honsu mega-earthquakes. In these studies, the F-T distribution $P(>T)$ were all nicely fitted with a one-dimensional $q$-exponential distribution of the form (4).

\subsection{NESP Formulation of Joint Distribution Laws}

We assume that the magnitude $M$, interevent time $\Delta t$ distributions are due to independent processes in the sense that the joint probability $P(M \cup \Delta t)$ factorizes into the probabilities of $M$ and $\Delta t$, i.e. $P(M \cup \Delta t)=P(M) P(\Delta t)$. Then, on removing the normalization, the joint probability above a magnitude threshold can be expressed by

$\log N=\log \left(N_{M=0}\right)+\left(\frac{2-q_{M}}{1-q_{M}}\right) \cdot \log \left(1-\frac{1-q_{M}}{2-q_{M}} \cdot \frac{10^{M}}{\alpha^{2 / 3}}\right)+\frac{1}{1-q_{t}} \log \left[1-\left(1-q_{t}\right)\left(\frac{\Delta t}{\Delta t_{0}}\right)\right]$,

where $q_{M}, q_{\mathrm{t}}$ are the entropic indices for the magnitude and interevent times respectively and $\Delta t_{0}$, is the q-relaxation time, analogous to the relaxation (characteristic) time often encountered in the analysis of physical systems. Eq. 6 comprises a generalized (bivariate) Gutenberg - Richter law and is the general model to be implemented in the ensuing analysis.

\section{Data Analysis}

\subsection{The North California Earthquake Catalogue}

The earthquake data utilized in this study was extracted from the regional earthquake catalogue compiled by the USGS/Northern California Seismic Network and available through the Northern California Earthquake Data Centre (NCEDC @ http://www.ncedc.org); it comprises 13165 events recorded within the area $35^{\circ} \mathrm{N} / 42^{\circ} \mathrm{N}$ and $234^{\circ} \mathrm{E} / 242 \mathrm{E}^{\circ} \mathrm{E}$ during 1967-2012.

Most earthquakes are reported in the local $\left(M_{L}\right)$ and moment $\left(M_{w}\right)$ magnitude scales, while there is a considerable number of events reported in the duration $\left(M_{d}\right)$ and amplitude $\left(M_{x}\right)$ scales. The latter have been exhaustively calibrated against the $M_{L}$ magnitude scale: Eaton (1992) has shown that they are within $5 \%$ of the $M_{L}$ scale for magnitudes in the range 0.5 to 5.5 and that they are virtually independent of the distance from the epicentre to at least $800 \mathrm{~km}$. Thus, $M_{d}$ and $M_{x}$ are practically equivalent to $M_{L}$. For the purpose of the present analysis the $M_{w}$ magnitudes were also converted to $M_{L}$ using the empirical formula of Urhammer et al (1996): $M_{w}=M_{L} \cdot(0.997 \pm 0.020)-(0.050 \pm$ 
0.131). Thus, the entire catalogue has been reduced to a proxy- $M_{L}$ scale. The NCEDC catalogue is practically homogeneous by construction and complete for $M_{L} \geq 3$, as demonstrated in Figure 1c.
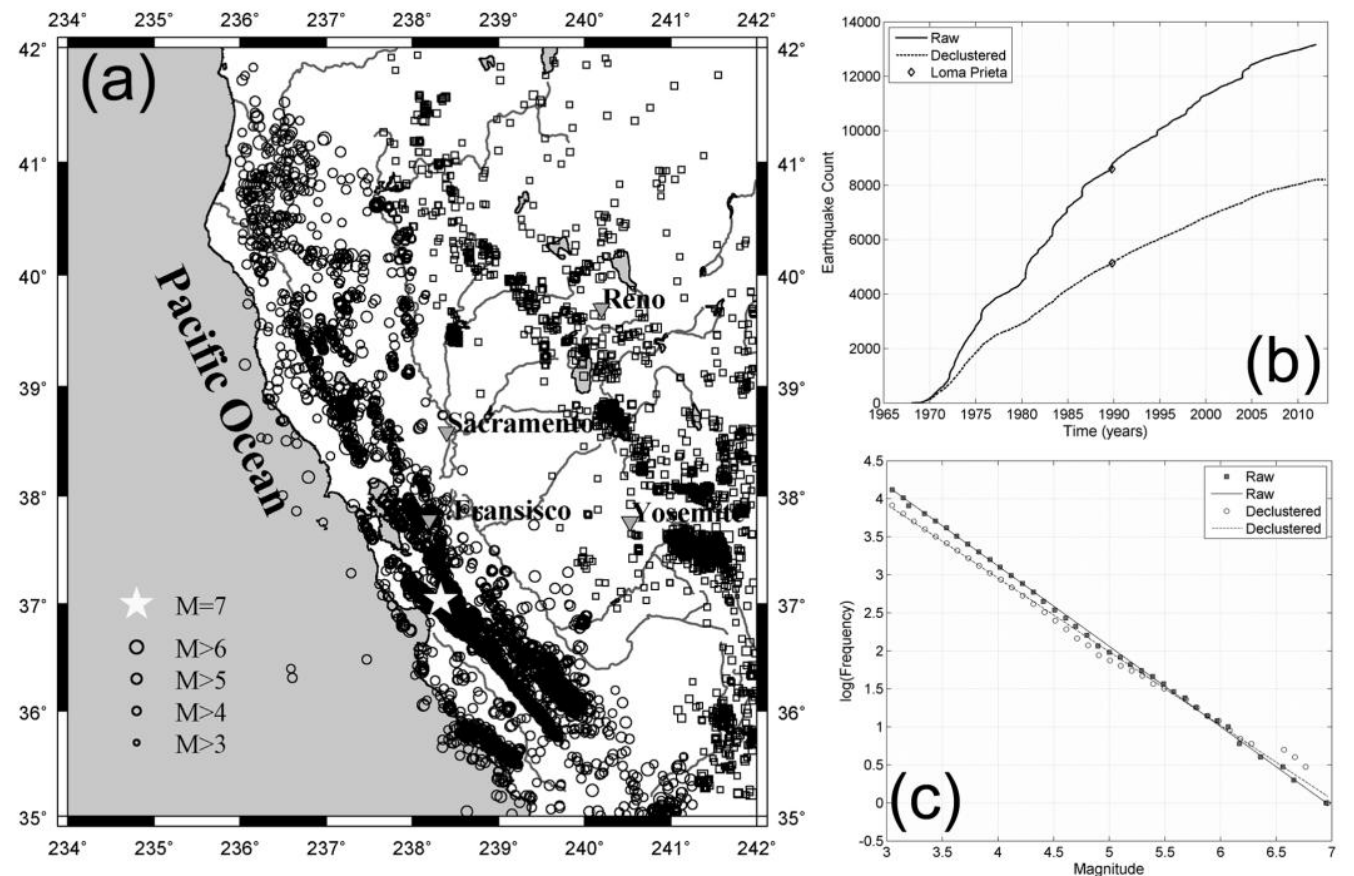

Figure 1 - The NCEDC catalogue, 1968-2012: (a) Epicentral distribution of the raw catalogue. (b) Cumulative earthquake counts of the raw and declustered catalogues. (c) The cumulative F-M distributions of the raw and declustered catalogues.

As stated in the introduction, there is an ongoing debate about the nature of interevent time frequency distributions with respect to whether they are characteristic and universal, or simply mixtures of correlated aftershock and uncorrelated background processes. This does not yet include the NESP formulation, but it is relevant nevertheless. In order to address this problem and compare the application of the NESP formalism to "mixed" and "background" processes, we have compiled a de-clustered version of the NCEDC catalogue using the approach of Reasenberg (1985). The declustered catalogue consists of 8210 events and its associated cumulative earthquake count is smooth and free of the time-local rate changes (jerks) evident in the corresponding curve of the raw catalogue due to aftershocks (Figure 1b); Consequently, it comprises mainly "background" earthquake activity; it is also homogeneous by construction and complete for $M_{L} \geq 3$ (Figure 1c).

\subsection{Empirical Cumulative Frequency Distributions and Probabilities.}

The joint F-M-T distribution is relatively easy to construct. A cut-off magnitude $M_{c}$ is set and a bivariate frequency table (histogram) representing the incremental distribution is first compiled; the cumulative frequency distribution is then obtained from the incremental distribution by backward bivariate summation. A typical example is shown in Figures $2 \mathrm{a}$ and $2 \mathrm{~b}$, of the empirical F-M-T distribution compiled from the raw NCEDC catalogue for $M_{c} \geq 3$.4. It is apparent that it is a well defined and structured surface, with its end-member at $\left[M \geq M_{c}, \Delta t=0\right]$ comprising the onedimensional Gutenberg - Richter law. The opposite end member located at $\left[M_{c}=0, \Delta t\right]$ comprises the one-dimensional F-T distribution. Both end-members of the distribution exhibit definite attributes of power-law dependence. In the above example, the cumulative frequency (earthquake count) can be written thus: $N\left(\left\{M \geq M_{c}, \Delta t: M \geq M_{c}\right\}\right)$. Then, the empirical probability $P\left(>\left\{M \geq M_{c}\right.\right.$, 
$\left.\left.\Delta t: M \geq M_{c}\right\}\right)$ is simply $\frac{N\left(>\left\{M \geq M_{c}, \Delta t: M \geq M_{c}\right\}\right)}{N\left(M_{c}, 0\right)}$, and $N\left(M_{c}, 0\right)=\|N\|_{\infty}$.

\subsection{Modelling Procedure}

The empirical F-M-T distributions of the form shown in Figure $2 b$, were approximated with the model of Eq. 6 using non-linear least-squares solver. Because the parameters of Eq. 6 are subject to positivity constraints and/or are bounded (e.g. the entropic indices), a solver implementing the trust-region reflective algorithm (e.g. Moré and Sorensen, 1983; Steihaug, 1983) was chosen, together with Least Absolute Residual minimization so as to down-weight possible outliers. A typical example is shown in Figure 2c and 2d. The quality of the approximation is exceptional, with the correlation coefficient $\left(R^{2}\right)$ being as high as 0.99 . The evaluation of the result is summarized in Figure 2d and is based on the analysis of the statistical distribution of the residuals. Thus, the observed cumulative probability of the sorted residuals is numerically approximated with a normal location-scale distribution (dashed line) and a Student-t location-scale distribution (solid line). Evidently, the residuals are not normally distributed and exhibit tails that are better approximated with the t-location-scale distribution. However, only 25 residuals deviate from the normal distribution, out of 173 , or $14.45 \%$; these represent outliers that have been effectively suppressed, since the solution is determined by the remaining $85.5 \%$ of the observations. It can also be seen that only 8 residuals are greater than $|0.5|$, or 3.16 earthquakes; these are, more or less scattered at the tails of the observed F-M-T distribution. Such statistics amply explain the quality of the solution and because they are typical, they strongly indicate that the NESP model represented by Eq. 6 is a "natural" description of earthquake (thermo)dynamics.
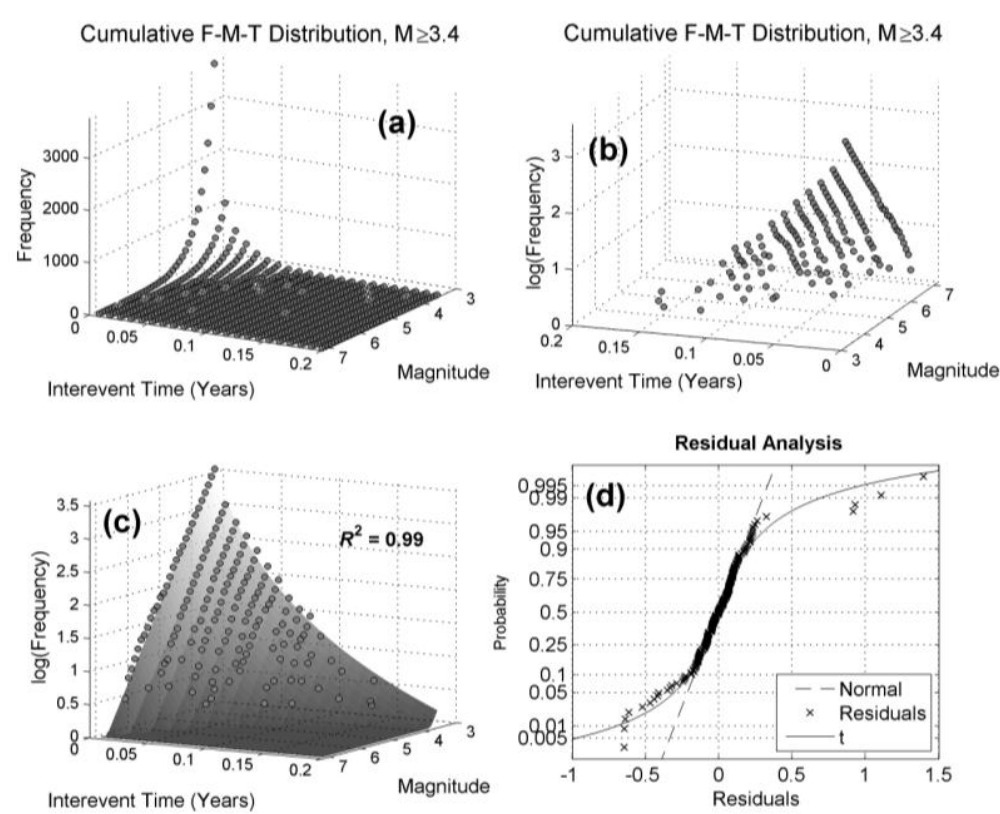

Figure 2 - (a) is the F-M-T distribution for the raw NCEDC catalogue and a cut-off magnitude of $M_{c} \geq 3.4$; (b) the same F-M-T but in logarithmic F-scale; (c) is as per (b) but with the predicted (fitted) distribution based on Eq. 6; (d) is a probability analysis of the residuals.

\section{Results}

As mentioned previously, the complexity and self-organization considered to characterize the seismogenetic system are associated with long-range interactions and long-term memory; these 
properties are collectively referred to as "correlation". The NESP theory proposes that the entropic indices are a measure of such correlation: values approaching unity indicate low correlation (near randomness) and vice versa.

As a first step in investigating such correlations we apply the analysis described in Section 3.3, to F-M-T distributions compiled from the raw and declustered NCEDC catalogues for different cutoff magnitudes. The results are presented in Figure 3. Excellent approximations were generally obtained, the least-squares goodness of fit $\left(R^{2}\right)$ being invariably higher than 0.93 . In consequence, and for the sake of experimental vigour, this presentation will only discuss results obtained from empirical F-M-T distributions constructed with more than 300 earthquakes and associated with a goodness of fit better than 0.97 .

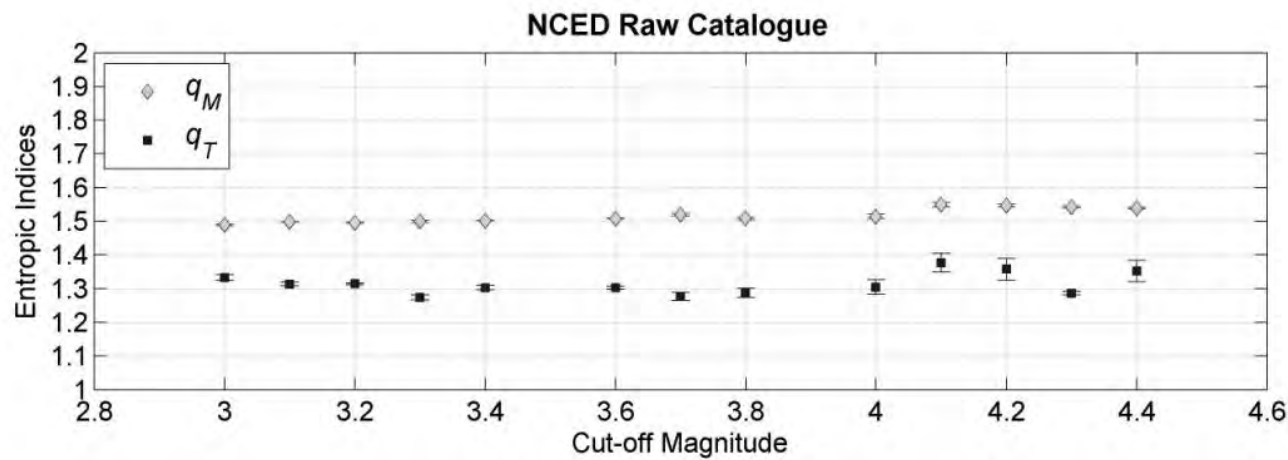

Figure 3 - The dependence of entropic indices estimated from the raw NCEDC catalogue on cut-off magnitudes. In all cases, error bars correspond to $95 \%$ confidence intervals

The raw NCEDC catalogue yields very stable $q_{\mathrm{M}}$ for all cut-off magnitudes, which varies from 1.49 at $M_{c}=3.0$ to 1.53 at $M_{c}=4.4$. It has been shown (e.g. Telesca, 2012) that Eq. 5 reduces to the conventional Gutenberg - Richter law and yields a proxy- $b$ value expressed in terms of the entropic index: $b_{q}=\left(2-q_{\mathrm{M}}\right) /\left(q_{M}-1\right)$. Application of this formula leads to proxy- $b$ value estimates that vary linearly from $\approx 1$ at $M_{c}=3.0$ to 0.88 at $M_{c}=4.4$ and can be verified to be consistent with corresponding determinations of $b$-values based on conventional methods. The entropic index $q_{M}$, like the $b$-value, represents the scaling of the size distribution of earthquakes and clearly indicates a correlated, scale-free process. Moreover, its small but persistent shift at the larger cutoff magnitudes indicates a corresponding increase in the size distribution of seismicity as a function of magnitude, albeit still correlated and scale-free. The temporal entropic index $q_{T}$ exhibits small variation with magnitude: it indicates low to moderate correlation for $M_{c} \leq 3.8$, where $q_{T} \approx 1.3$, rising towards 1.4 thereafter (moderate correlation).

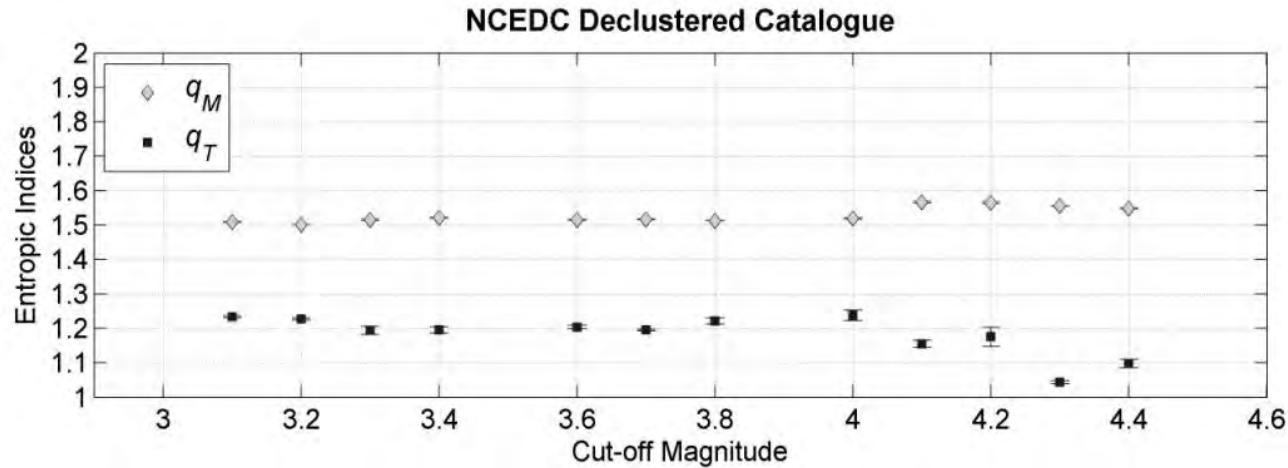

Figure 4 - As per Figure 3 for the declustered NCEDC catalogue.

XLVII, No $3-1333$ 
The analysis of the declustered NCEDC catalogue is shown in Figure 4. $q_{M}$ varies quasi-linearly from 1.5 at $M_{c}=3$ to and 1.53 at $M_{c}=4.4$ and is consistent with the corresponding index derived from the raw catalogue. This yields proxy- $b$ values also quasi-linearly changing from 1 at $M_{c}=3$ to 0.88 at $M_{c}=4.4$, and is generally consistent with conventional determinations of $b$. Here as well, the small bt persistent shift of $q_{M}$ at the larger cut-off magnitudes indicates a corresponding change in the size distribution of background earthquakes almost identical to that of the mixed background aftershock process. The temporal entropic index $q_{T}$, however, behaves almost exactly opposite to its raw-catalogue counterpart: it is approximately equal to 1.2 for $M_{c}=\leq 4$ (low correlation) and gradually drops to 1.1 at $M_{c}=4.4$, which indicates very weak correlation or, equivalently, a practically random temporal sequence of earthquake occurrence, at least for cut-off magnitudes of the order of $4.2-4.3$.

The comparison of the results from the raw and declustered catalogues shows that the size distribution of background earthquake processes on one hand, and mixed background - aftershock processes on the other, are compatibly correlated in a way consistent with the phenomenology expected from the SOC theory (e.g. Bak et al., 2002). Conversely, the temporal sequence of background earthquakes appears to be weakly correlated or random at worst, while the temporal sequence of the mixed process appears to be moderately correlated. At first sight, this would appear to be consistent with the model-based studies of Touati et al (2009). It is worth noting, however, that the moderate - good temporal correlation of the mixed process persists at $M_{c} \geq 4$ Although these results cannot be light-heartedly extrapolated to larger magnitude scales, the observation may suggest that at intermediate magnitude scales, aftershocks and background events are entangled and interact, thus producing SOC phenomenology in the temporal expression of regional seismicity. According to this view, aftershocks may be an integral part of the regional seismicity process and not just localized peculiarities (also see Marsan and Lengliné, 2008).
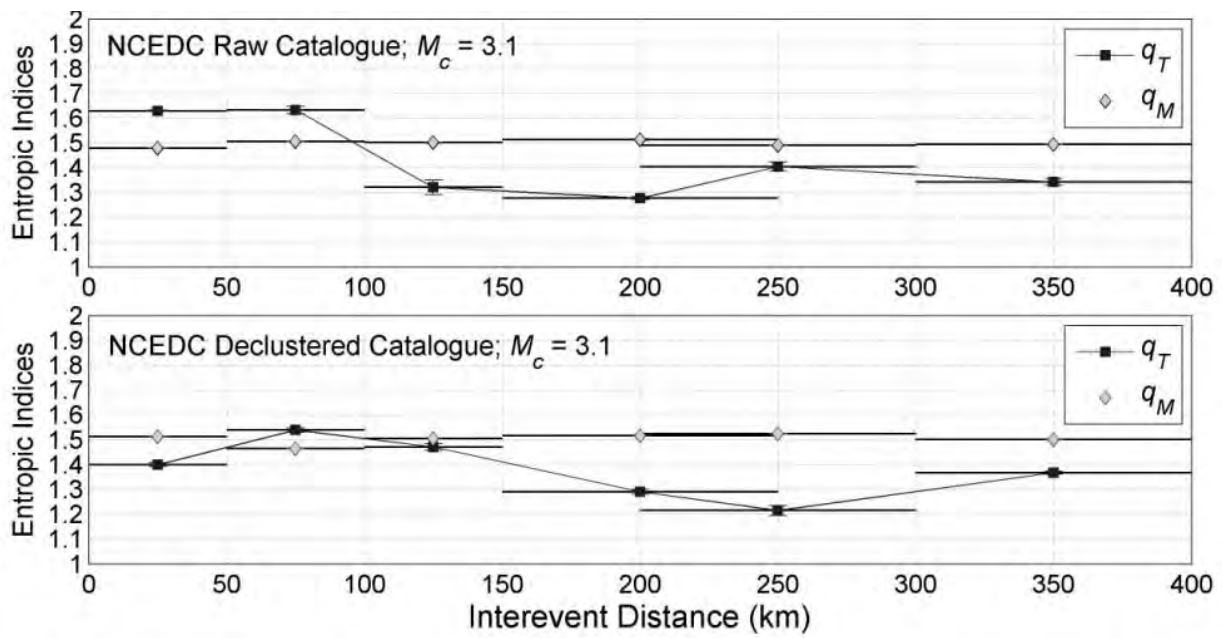

Figure 5 - The dependence of the entropic indices on interevent distance, for data subsets grouped according to interevent distance. (a) Analysis of the raw catalogue. (b) Analysis of the declustered catalogue.. The extent of $\Delta d$ bins is indicated by horizontal line segments. Errors are $95 \%$ conf. intervals.

Next, we study the effect of spatial separation (interevent distance) on earthquake size distribution and temporal expression. The modelling procedure was applied to data subsets grouped according to the interevent distance between consecutive events, following the rule $C \supset\left\{C_{D}: M>M_{c} \wedge \Delta d_{L}\right.$ $\left.\leq \Delta d \leq \Delta d_{U}\right\}$ where $C$ is the catalogue, $C_{D}$ is the subset catalogue, $\Delta d$ is the interevent distance and $\Delta d_{L}, \Delta d_{U}$ are the upper and lower group limits. The resulting variation of the entropic indices with respect to distance group is shown in Figure 5 for both the raw (top panel) and declustered (bottom 
panel) catalogues. The breadth of the interevent distance bins is indicated by the horizontal line segments across the index symbols. The results have been derived for $M_{c}=3.1$.

The analysis of the raw NCEDC catalogue (Figure 5a) shows that $q_{M}$ is consistently determined as a function of interevent distance. It can be seen to vary between 1.46 and 1.52 with the corresponding proxy $b$-values varying between 1.17 and 0.92 . The temporal entropic index $q_{T}$ exhibits significant variation with increasing interevent distance group. It begins by showing a very highly correlated process $\left(q_{T} \approx 1.6\right.$ at $\left.\Delta d=0-100 \mathrm{~km}\right)$, which is evidently due to the overwhelming effect of the tightly spaced and highly correlated aftershock sequences. At longer interevent distances $q_{T}$ drops to $1.3-1.4$ (moderate-good correlation). The high correlation at the short(er) interevent distances due to the tightly spaced aftershock sequences should have been expected, but the interesting observation here is that the time dependence of earthquake occurrence in the mixed background-and-aftershock process is correlated over long distances, implying the existence of long range interactions in the mixed process.

The analysis of the declustered NCEDC catalogue is shown in Figure 5b. The magnitude entropic index $q_{M}$ behaves in a manner similar to its raw counterpart. It can be seen to vary between 1.48 and 1.52 , with the corresponding proxy $b$-values varying between 1.08 and 0.92 . The temporal entropic index $q_{T}$ begins with a correlated process $\left(q_{T}=1.41-1.52\right.$ at $\left.\Delta d<150 \mathrm{~km}\right)$, which is attributed to near field interaction between nearby earthquake pairs and at longer interevent distances drops to $1.2-1.37$ (low - moderate correlation).

\section{Discussion and Conclusions}

The present work marks the beginning of a systematic attempt to test this proposition: Insofar as earthquakes occur as manifestations of a complex non-extensive seismogenetic system, their frequency of occurrence should be related to the magnitude, the interevent time and the interevent distance by multidimensional power-laws consistent with the formalism of Non-Extensive Statistical Physics (NESP). The problem is addressed by constructing multivariate empirical earthquake frequency distributions with respect to the parameters quoted above, rigorously approximating them with theoretical models based on the NESP formalism and evaluating the results relative to the established ken on earthquake statistics. The case study reported herein is focusing on aspects of North Californian (USA) seismicity.

The results indicate that NESP is an excellent descriptor of the statistical physics of earthquakes. Provided that the multivariate empirical frequency distributions are based on a sufficient number of observations - a few hundred is an empirical lower limit - the results are stable and consistent with the established ken, irrespective of the magnitude and spatio-temporal range of the earthquake catalogue, or operations pertaining to re-sampling, bootstrapping or re-arrangement of the catalogue. In this sense the NESP formalism appears to apply to Northern Californian earthquake statistics and with time and due testing, may prove to comprise a universal descriptor of seismicity.

With respect to Northern Californian seismicity, the analysis of the size (energy) distribution of earthquakes yielded results consistent with a correlated sub-extensive system; the results are also compatible with observations based on conventional measures of scaling and self-organization, with particular reference to the $b$-value. There is also evidence for variation in the size distribution: the scaling of earthquake sizes appears to decrease for cut-off magnitudes larger than four, albeit by a very small factor. At this point, however, it cannot be positively determined whether this is a natural phenomenon, peculiarity of the catalogue, or a statistical/modelling artefact; the existing evidence is limited and does not warrant additional discussion and inference.

The analysis of interevent times, i.e. the time dependence of earthquake occurrence, has determined the existence of sub-extensivity and near-field interaction (correlation) in the complete record of North Californian seismicity (the raw catalogue comprising of mixed background

XLVII, No 3 - 1335 
earthquake activity and aftershock processes), as well as in the background process alone (declustered catalogue). The former is attributed to the joint effect of near-field interaction between neighbouring earthquakes or seismic areas and interaction within the tightly spaced and correlated aftershock sequences. The latter is attributed to near-field interaction only. Good farfield correlation (between distant earthquake pairs) was detected only in the mixed process; which also yields evidence of the correlation strengthening for cut-off magnitudes larger than four. The background process appears to be moderately - weakly correlated at the far field. Properly random temporal processes $\left(q_{T} \approx 1\right)$ have not been detected.

A general syllogism affordable by the above observations is that aftershock sequences may be an integral part of the seismogenetic process, as they appear to partake in long-range interaction. A formal explanation of such an effect is pending, but may nevertheless involve delayed remote triggering of seismic activity by (transient or static) stress transfer from the main shocks and large aftershocks and/or cascading effects already discussed by Marsan and Lengliné (2008). In this view, the effect weakens when aftershocks are removed because aftershocks are the link between the main shocks and their remote offshoot.

As a general conclusion, it may be stated that the expression of seismicity at Northern California is generally consistent with non-extensive (sub-extensive) thermodynamics. The NESP formalism, although far from having settled the questions and debates on the statistical physics of earthquakes, appears, nevertheless, to be an effective and insightful tool in the investigation of seismicity and its associated complexity.

\section{Acknowledgments}

This work was supported by the THALES Program of the Ministry of Education of Greece and the European Union in the framework of the project "Integrated understanding of Seismicity, using innovative methodologies of Fracture Mechanics along with Earthquake and Non-Extensive Statistical Physics - Application to the geodynamic system of the Hellenic Arc - SEISMO FEAR HELLARC".

\section{References}

Abe S. and N. Suzuki 2005. Scale-free statistics of time interval between successive earthquakes, Physica A, 350, 588-596.

Abe S., Suzuki N. 2003. Law for the distance between successive earthquakes. J. Geophys. Res., 108 (B2), 2113.

Baiesi M. and Paczucki M. 2004. Scale-free networks of earthquakes and aftershocks, Phys. Rev. $E, 69,066106$; doi: 10.1103/PhysRevE.69.066106.

Bak P. and C. Tang 1989. Earthquakes as a self-organized critical phenomenon. J. Geophys. Res., 94, 15635-15637.

Bak P., Christensen K., Danon L. and T. Scanlon 2002. Unified Scaling Law for Earthquakes. Phys. Rev. Lett., 88, 178501.

Carbone V., Sorriso-Valvo L., Harabaglia P., Guerra I., 2005. Unified scaling law for waiting times between seismic events, Europhys. Lett., 71 (6), p. 1036 (2005) doi: $10.1209 / \mathrm{epl} / \mathrm{i} 2005-10185-0$

Celikoglu A., Tirnakli U., and Duarte Queir'os S., 2010: Analysis of return distributions in the coherent noise model, Phys. Rev. E, 82, 021124, doi:10.1103/PhysRevE.82.021124.

Corral A. 2004. Long-Term Clustering, Scaling, and Universality in the Temporal Occurrence of Earthquakes, Phys. Rev. Lett., 92 (10), 108501; DOI: 10.1103/PhysRevLett.92.108501

Eaton J.P. 1992. Determination of amplitude and duration magnitudes and site residuals from short-period seismographs in Northern California. Bull. Seism. Soc. Am., 82 (2), 533-579.

Gell-Mann M. and Tsallis C. (eds.), 2004. Nonextensive Entropy - Interdisciplinary Applications. Oxford University Press, New York.

XLVII, No $3-1336$ 
Gutenberg B. and Richter C.F. 1944. Frequency of earthquakes in California, Bull. Seismol. Soc. Am., 34-4, 185-188.

Marsan D. and Lengliné O. 2008. Extending earthquakes's reach through cascading, Science, 319, 1076; doi: 10.1126/science.1148783

Moré J.J. and Sorensen D.C. 1983. Computing a Trust Region Step, SIAM Journal on Scientific and Statistical Computing, 3, 553-572.

Ogata, Y. 1988. Statistical models for earthquake occurrences and residual analysis for point processes, J. Am. Statist. Assoc., 83, 9-27.

Reasenberg P. 1985. Second-order moment of central California seismicity, 1969-82, J. Geophys. Res., 90, 5479

Saichev A. and Sornette D. 2012. Fertility Heterogeneity as a Mechanism for Power Law Distributions of Recurrence Times. arXiv:1211.6062, available online at http://arxiv.org/abs /1211.6062v1 (last access 31 January 2013).

Silva R., Franca G.S., Vilar C.S., Alcaniz J.,S., 2006. Nonextensive models for earthquakes. Physical Review E, 73, 026102. doi:10.1103/PhysRevE.73.026102.

Sornette A. and Sornette D. 1989. Self-organized criticality and earthquakes, Europhys. Lett., 9, 197-202.

Sotolongo-Costa O. and Posadas A. 2004. Tsalli's entropy: A non-extensive frequency-magnitude distribution of earthquakes, Phys. Rev. Letters, 92 (4), 048501; doi:10.1103/PhysRevLett.92 .048501 .

Steihaug T. 1983. The Conjugate Gradient Method and Trust Regions in Large Scale Optimization, SIAM Journal on Numerical Analysis, 20, 626-637.

Telesca L. 2011. Tsallis-based nonextensive analysis of the Southern California seismicity. Entropy, 13, 1267-1280.

Telesca L. 2012. Maximum Likelihood Estimation of the Nonextensive Parameters of the Earthquake Cumulative Magnitude Distribution, Bull. Seismol. Soc. Am., 102, 886-891.

Touati S., Naylor M. and Main I.G., 2009. Origin and Nonuniversality of the Earthquake Interevent Time Distribution. Phys. Rev. Letters, 102, 168501; doi: 10.1103/ PhysRevLett.102.168501

Tsallis C. 1988. Possible generalization of Boltzmann-Gibbs statistics. Journal of Statistical Physics, 52: 479-487. doi:10.1007/BF01016429.

Tsallis C. 2009. Introduction to Nonextensive Statistical Mechanics: Approaching a Complex World. Springer Verlag, Berlin, 378pp.

Uhrhammer B. R. A., Loper S. J., and Romanowicz B., 1996. Determination of local magnitude using BDSN Broadband Records, Bull. Seism. Soc. Am., 86 (5), 1314-1330.

Vallianatos F. and Sammonds P. 2013. Evidence of non-extensive statistical physics of the lithospheric instability approaching the 2004 Sumatran-Andaman and 2011 Honshu megaearthquakes, Tectonophysics (2013), doi: 10.1016/j.tecto.2013.01.009.

Vallianatos F., Michas G., Papadakis G. and Tzanis A., 2013. Evidence of non-extensivity in the seismicity observed during the 2011-2012 unrest at the Santorini volcanic complex, Greece, Nat. Hazards Earth Syst. Sci., 13, 177-185; doi:10.5194/nhess-13-177-2013.

Vallianatos F., Michas G., Papadakis G., Sammonds P., 2012. A non-extensive statistical physics view to the spatiotemporal properties of the June 1995, Aigion earthquake (M6.2) aftershock sequence (West Corinth rift, Greece). Acta Geophysica, 60, 3, 758-768.

Vallianatos F. and Telesca L. (Eds.), 2012.. Statistical Mechanics in Earth Physics and Natural Hazards, Acta Geophysica, 60, 499-501. 